\title{
Opportunities for collaboration on imaging development in NCI
}

\section{programs}

\section{Overview of NCI's Cancer Imaging Program}

Daniel C. Sullivan

National Cancer Institute, Cancer Imaging Program, Rockville, MD 20852, USA

http://imaging.cancer.gov

\section{Mission}

The mission of the Cancer Imaging Program (CIP), National Cancer Institute, is to promote and support:

- Cancer-related basic, translational and clinical research in imaging sciences and technology, and

- Integration and application of these imaging discoveries and developments to the understanding of cancer biology and to the clinical management of cancer and cancer risk.

\section{CIP Vision and Goals}

Cancer Imaging: visualize the problem and direct the solution.

1. Infrastructure and programs to support the discovery and development of molecular imaging for cancer care and understanding of cancer biology.

2. A set of imaging methods validated as cancer biomarkers, some of which are surrogate endpoints.

3. Infrastructure and programs to support the discovery, development and delivery of imagedependent interventions for cancer and precancer.

4. An implemented infrastructure based on standardized models for the design and conduct of clinical trials of, or using, imaging and imageguided interventions.
5. Accelerated development and delivery of integrated imaging systems and methods for cancer care and research.

6. Critical role in NIH and NCI activities in emerging technologies, such as nanotechnology, proteomics, and high-throughput screening technologies.

7. An implemented informatics infrastructure to optimize the value of cancer imaging data.

8. A strategy of imaging science and methods to detect, treat and monitor response to therapy in pre-cancer.

\section{Overview of NCI's Specialized Programs of Re- search Excellence program}

Jorge Gomez

National Cancer Institute, Organ Systems Branch, Office Centers Training and Resources, Rockville, MD 20852, USA

http://spores.nci.nih.gov

Specialized Programs of Research Excellence (SPOREs) are funded through specialized center grants (P50s) that promote interdisciplinary research and move basic research findings from the laboratory to clinical settings, involving both cancer patients and populations at risk of cancer. The outcome of interdisciplinary research is a bidirectional approach to translational research, moving laboratory discoveries to clinical settings or clinical observations to the laboratory environment. Laboratory and clinical scientists share the common goal of bringing novel ideas to clinical care settings that have the potential to reduce cancer incidence and mortality as well as improve survival and the quality of life. In order to achieve these goals, SPORE investigators work collaboratively to plan, design, and implement research programs that may impact cancer 
prevention, detection, diagnosis, and treatment. Additionally, SPOREs approach these goals through collaborative efforts within the individual multidisciplinary SPORE teams, inter-SPORE collaborations, partnerships with other NCI/NIH programs, and public-private partnerships with industry and non-profit organizations. Key qualities of the program feature the inclusion of patient advocates in SPORE activities and international cooperation with investigators in Europe, Canada, Asia, and Mexico.

\section{Overview of NCI's Division of Cancer Prevention Early Detection Research Network}

Sudhir Srivastava

National Cancer Institute, Cancer Biomarkers Research Group, Rockville, MD 20852, USA

http://edrn.nci.nih.gov

The Early Detection Research Network (EDRN), an initiative of NCI, brings together dozens of institutions to help accelerate the translation of biomarker information into clinical applications and to evaluate new ways of testing cancer in its earliest stages and for cancer risk.

EDRN offers investigators outside of the Network opportunities for collaboration as Associate Members in research efforts related to biomarker development, clinical testing and validation. Non-EDRN investigators can develop informal or formal collaborative research through an EDRN Principal Investigator, or through the EDRN Collaborative Groups, and request the use of shared resources such as novel technologies, specimens, high-risk registries, and cohorts through the Network, or seek funding for validation studies.

The components and projects that comprise the Early Detection Research Network:

\section{- Biomarker Development Laboratories}

Responsible for the development and characterization of new biomarkers or the refinement of existing biomarkers.

- Biomarker Reference Laboratories

Serve as a Network resource for clinical and laboratory validation of biomarkers, which includes technological development, quality control, refinement, and high throughput.

- Clinical Epidemiology and Validation Centers Conduct clinical and epidemiological research regarding the clinical application of biomarkers.

\section{- Data Management and Coordinating Center}

Coordinates the EDRN research activities, providing logistic support, and conducting statistical and computational research for data analysis, analyzing data for validation. Also responsible for EDRN common database development.

- Informatics Center

The Informatics Center supports EDRN's efforts through the development of software systems for information management. The center is at the NASA Jet Propulsion Laboratory at the California Institute of Technology.

Overview of NCI's Division of Cancer Prevention Phase II Chemoprevention Clinical Trial Consortia

Eva Szabo

National Cancer Institute, Lung \& Upper Aerodigestive Cancer Research Group, Rockville, MD 20852, USA

http://www.cancer.gov/newscenter/pressreleases/ preventtrials

NCI, DCP has created a consortium of research centers to conduct early-phase cancer prevention clinical trials. Six institutions have been chosen to undertake these critical studies to assess the cancer preventive potential of new agents.

The consortium members:

- Design and conduct early-phase clinical trials to assess the cancer prevention potential of a variety of agents, many of which target specific molecules known to be expressed in precancerous conditions.

- Characterize the effects of these agents on endpoints associated with cancer development (such as cell proliferation, apoptosis, growth factor expression, oncogene expression, and additional markers) and correlate these effects with clinical endpoints.

- Develop scientific insights into the mechanisms of cancer prevention by assessing the clinical effects of these candidate agents, and by testing novel markers that may be used to determine response to the agents.

- Each consortium member collaborates with a network of institutions to conduct these studies and to recruit study participants. The consortium members design and implement numerous studies on agents that may play a role in preventing cancer. 


\section{Overview of NCI's Translational Research Working Group}

\section{Ernest T. Hawk}

National Cancer Institute, Office of Centers for Training \& Resources, Rockville, MD 20852, USA

http://www.cancer.gov/trwg

The Translational Research Working Group (TRWG) was established in the summer of 2005 to conduct a discussion with the broader cancer research community to develop recommendations about how NCI can best organize its investment to further "translational research."

Over the next year, the TRWG will review NCI's current intramural and extramural translational research portfolio (within the scope of the TRWG mission), facilitate broad community input, invite public comment, and recommend ways to improve and integrate efforts. The ultimate goal is to accelerate progress toward improving the health of the nation and cancer patient outcomes.

The TRWG plans to accomplish its mission using the following phases:

- Phase 1: First, the TRWG will evaluate the current portfolio of NCI-funded translational acti- vities and invite input from the broad cancer community and the general public about the strengths of that portfolio as well as ways to improve and enhance its focus, integration, and effectiveness.

- Phase 2: Second, the TRWG will devise an optimized translational research model and offer recommendations about how NCI can achieve this future vision for translational research. The TRWG will share these recommendations with the cancer community and again invite public input.

- Phase 3: Finally, the TRWG will finalize its model and recommendations, and will develop an implementation plan. The plan may include both shortterm adjustments intended to better harmonize existing programs, as well as long-term initiatives that may transcend existing programs.

\section{NCI's commitment to translational research}

NCI is committed to speeding the development of new diagnostic tests, cancer treatments, and other interventions that benefit people with cancer and people at risk for cancer. Such development relies on strong translational research collaborations between basic and clinical scientists to generate novel approaches 